\title{
Comprehensive review of tribometer dynamics-Cycle-based data analysis and visualization
}

\author{
Josef PROST ${ }^{*}$, Guido BOIDI, Thomas LEBERSORGER, Markus VARGA, Georg VORLAUFER \\ AC2T research GmbH, Wiener Neustadt 2700, Austria \\ Received: 18 December 2020 / Revised: 09 April 2021 / Accepted: 03 June 2021 \\ (C) The author(s) 2021.
}

\begin{abstract}
Tribologists often rely on triboexperiments to investigate factors that affect a tribosystem. The inherent dynamic behavior of the respective tribometer setups and its effect on data interpretation remain often unknown. In this study, a comprehensive analysis of sensor data obtained from lubricated and dry triboexperiments is performed. Data are generated on a pin-on-disc test rig with a silicon nitride ball on a steel disc contact with a rotation frequency of $\sim 3 \mathrm{~Hz}$. High-speed acquisition of sensor data up to $5 \mathrm{kHz}$ is performed to resolve changes in the data within individual cycles. The characteristic frequencies of the system and their temporal evolution are determined via time-frequency analysis, which reveals periodic patterns in the sensor data. Cycle-based data evaluation allows the detection of localized events and changes during an operation and considerably reduces the apparent measurement uncertainty, as compared with an unreduced dataset. The data analysis and visualization routines presented herein may serve as a prototype for further application to tribometer setups.
\end{abstract}

Keywords: high-speed data acquisition; time-frequency analysis (TFA); cycle-resolved analysis; pin-on-disc tribometer

\section{Introduction}

The readily available high-speed and high-volume storage technology enables the time-series data of sensor signals to be digitally recorded with an extremely high temporal resolution for the full duration of a tribometrical experiment. Subsequently, these data can be numerically processed to investigate dynamical effects, such as friction-induced vibrations [1, 2] or the transition from static to dynamic friction [3]. Consequently, time- and location-resolved friction information, which is the target of this study, can be obtained.

A pin-on-disc tribometer is a prototypical model tribometer setup used for simulating sliding contacts; it comprises a stationary pin pressed against the flat side of a rotating disc. For this setup, a few standard test methods provided by international standard organizations, such as the American Society for Testing and Materials (ASTM) or the International Organization for Standardization (ISO), can be used, e.g., ASTM-99 [4] or ISO 7148-1 [5] and ISO 7148-2 [6]. It is a well-established method for characterizing lubricant properties [7-9], material pairings in dry contact $[10,11]$, or specific geometries [12, 13]. Typically, the disc is operated at a constant rotational speed. Theoretically, the pin-on-disc setup should provide stationary friction conditions; however, in practice, dynamic behaviors in tribology have been observed for many years [14] but rarely investigated [15-17]. The slightest misalignment between the nominal disc surface normal and the rotation axis or the waviness of the disc surface will cause a periodic motion of the pin in the loading direction, thereby resulting in a periodic change in the normal force. In addition, local variations in friction or lubrication

* Corresponding author: Josef PROST, E-mail: josef.prost@ac2t.at 


\begin{tabular}{|c|c|c|c|}
\hline \multicolumn{4}{|c|}{ Nomenclature } \\
\hline$\mu$ & Coefficient of friction & $r$ & Radius of apparent contact surface (mm) \\
\hline$A$ & Cross-sectional area of the wear profile & $r_{\text {track }}$ & Nominal track radius $(\mathrm{mm})$ \\
\hline & $\left(\mathrm{mm}^{2}\right)$ & $S_{x x}(f, \tau)$ & Spectral energy density of force sensor \\
\hline$d_{\max }$ & Maximum wear depth (mm) & & $\operatorname{signal}\left(\mathrm{N}^{2} \cdot \mathrm{s}^{2}\right)$ \\
\hline$F_{\mathrm{f}}$ & Friction force $(\mathrm{N})$ & $V_{\text {ball }}$ & Wear volume of ball $\left(\mathrm{mm}^{3}\right)$ \\
\hline$F_{\mathrm{N}}$ & Normal force $(\mathrm{N})$ & $V_{\text {disc }}$ & Wear volume on disc surface $\left(\mathrm{mm}^{3}\right)$ \\
\hline$h_{\text {ball }}$ & Wear height of ball (mm) & $w(t-\tau)$ & Windowing function \\
\hline$R$ & Radius of ball (mm) & $X(f, \tau)$ & STFT of a force sensor signal $(\mathrm{N} \cdot \mathrm{s})$ \\
\hline
\end{tabular}

conditions, such as those due to anisotropic roughness [18], may occur. Because many tribometer configurations involve a characteristic unidirectional or oscillating sliding motion with a typical frequency in the range of a few hertz, the analysis and visualization procedures described herein may be applicable or adapted to data obtained from similar setups.

Tribometer dynamics can be identified in sensor data using appropriate filtering techniques and can be segregated effectively from signal noise. In particular, in cases where only long-time transient behavior is considered, e.g., when only one average value of the coefficient of friction (CoF) is reported per stroke or cycle $[17,19,20]$, a meticulous analysis of time-resolved sensor data may increase the significance of the reported experimental results [21, 22].

Time-frequency analysis (TFA) [23-25] is an effective tool for analyzing quasi-periodic contributions in non-periodic time-series data. TFA is used for a wide range of applications, including tool condition monitoring [26], fault diagnostics in water pipelines [27], and machinery fault diagnostics [28]. Recently, the authors performed TFA to detect cold-induced noise emissions from porous journal bearings [29]. Previous studies pertaining to the application of TFA to tribological questions are limited to data obtained from non-standard sensors, such as acoustic emissions and laser Doppler vibrometers [30,31] or piezoelectrical vibration sensors [29]. The time-dependent investigation of wear mechanisms based on friction force signals performed by Song et al. [32] demonstrated the applicability of TFA to force sensor data.

In this study, a comprehensive numerical data analysis and visualization techniques were employed to investigate the machine-dynamic behavior of a pin-on-disc tribometer. The findings obtained were considered for further statistical data evaluation based on individual cycles. The procedures performed included outlier removal using robust nonlinear filters, smoothing using local polynomial regression filters, TFA, and three-dimensional (3D) graphical representation for convenient visual data exploration. The proposed procedures were compared with standard data evaluation methods to determine the additional possibilities provided by cycle-based data evaluation.

\section{Tribometer}

\subsection{Pin-on-disc tribometer and materials}

Frictional experiments were performed using a laboratory-built pin-on-disc test rig, as shown in Fig. 1. The counter body (either pin or ball samples) was loaded statically against a rotating disc driven by a motor. The rotational speed of the disc can be continuously varied between 10 and 3,000 rpm, corresponding to rotational frequencies from 0.17 to $50 \mathrm{~Hz}$.

Experiments were performed at a constant tangential speed of $1 \mathrm{~m} / \mathrm{s}$ (191 rpm on a $100 \mathrm{~mm}$ diameter, corresponding to $3.18 \mathrm{~Hz}$, Fig. 1(b)). A constant vertical load of $20 \mathrm{~N}$ was applied in all experiments by placing a static load on the loading lever. In the lubricated experiments, lubricant was deposited continuously via a sponge placed at the same level as the disc surface, as shown in Fig. 1(b). The sponge was impregnated based on the same procedure in all experiments and mounted in the same position, 


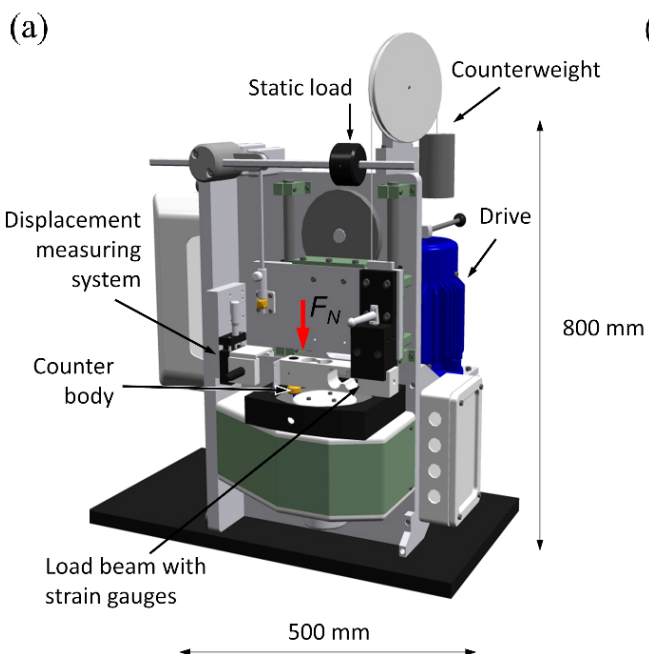

(b) Schematic top view

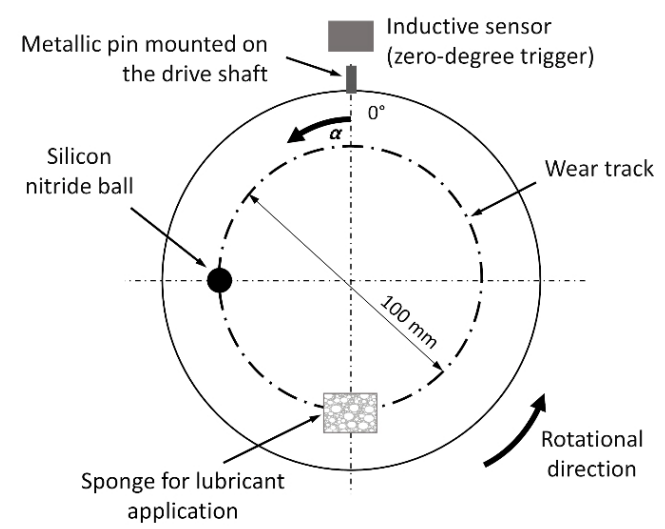

Fig. 1 (a) Schematic illustration of pin-on-disc test rig used in this study ( $F_{\mathrm{N}}$ is the applied normal load). (b) Schematic illustration of the setup from top view. $\alpha$ is the angular position on the wear track with respect to the zero-degree position defined by the trigger.

resulting in reproducible contact between the disc and sponge.

In the present study, additive-free paraffin oil (paraffin liquidum) was used in the lubricated experiments to avoid complexities due to lubricant addition, i.e., tribofilm formation. A silicon nitride bearing ball (hardness 1,466 \pm 14 HV1) with a diameter of $10 \mathrm{~mm}$ was pressed against a hardened bearing steel (100Cr6/AISI 52100; hardness $62 \mathrm{HRC} / 750 \mathrm{HV}$ ) disc with a diameter of $110 \mathrm{~mm}$ and a height of $10 \mathrm{~mm}$. The standard composition of 100Cr6 steel according to EN ISO 683-17 [33] is listed in Table 1. The bearing ball had a certified surface roughness $R a=40 \mathrm{~nm}$ (grade G25), whereas the steel discs were lapped with a standard custom-made procedure to yield $R a=20 \pm 5 \mathrm{~nm}$.

In total, 10 lubricated and 10 dry experiments were conducted. All experiments were performed in an air-conditioned laboratory at room temperature of $22{ }^{\circ} \mathrm{C}$ and an ambient relative humidity of $60 \%$. The duration of each experiment was set to $1 \mathrm{~h}$. The experimental parameters were selected to facilitate operations under hydrodynamic lubrication to achieve stable frictional behaviors during the experiment. The guaranteed stability of the system allowed cyclic variations caused by the intrinsic dynamics of the tribometer system to be scrutinized. Meanwhile, the dry experiments were significantly affected by the interactions between the asperities of the two rubbing bodies; therefore, a dynamical behavior was expected over time, i.e., the attainment of a steady operation state after an unstable run-in phase. These changes were reflected in the recorded sensor data.

\subsection{Data acquisition}

The tribometer was equipped with an in-house designed two-axis load beam and two full bridges of linear strain gauges (resistance of $120 \Omega$ [34]) for friction and normal force measurements, as shown in Fig. 1(a). The displacement of the load unit with respect to the reference frame was determined using a laser distance sensor [35] with a measurement accuracy of $1 \mu \mathrm{m}$.

The zero position of each rotating cycle was triggered using an inductive sensor activated by a metallic pin mounted on the drive shaft, as shown in Fig. 1(b).

The experiments were controlled using an in-house developed data acquisition and control software, which was also used to record the sensor data. The full bridges of the load beam were connected to an analog amplifier [36]. The output of the amplifier

Table 1 Standard composition of 100Cr6 steel according to EN ISO 683-17 [33].

\begin{tabular}{cccccccc}
\hline & $\mathrm{C}$ & $\mathrm{Si}$ & $\mathrm{Mn}$ & $\mathrm{P}$ & $\mathrm{S}$ & $\mathrm{Cr}$ & $\mathrm{Mo}$ \\
\hline$($ at $\%)$ & $0.93-1.05$ & $0.15-0.35$ & $0.25-0.45$ & $\leq 0.025$ & $\leq 0.015$ & $1.35-1.60$ & $\leq 0.10$ \\
\hline
\end{tabular}


and the sensors were connected to a high-speed data acquisition board, which allows to record up to 250,000 samples per second at a resolution of 16 bits, to accommodate the analog inputs [37]. The dry and lubricated experiments were performed with acquisition rates of 1 and $5 \mathrm{kHz}$, respectively, to increase the temporal resolution and accessible frequency range. According to the Shannon-Nyquist sampling theorem [38], the maximum detectable frequencies were 0.5 and $2.5 \mathrm{kHz}$ for the dry and lubricated experiments, respectively.

Data were stored in the HDF5 file format [39] to facilitate post-processing. In each HDF5 file, a second dataset with the sensor data downsampled to $10 \mathrm{~Hz}$ was stored. This downsampling was achieved using the means of 100 and 500 consecutive data points.

To extract angle-resolved information from the sensor data, the zero-position was triggered using an inductive sensor. Angular positions along the wear track, corresponding to angle $\alpha$ in Fig. 1(b), were calculated by linearly interpolating the space between two trigger signals into 360 equidistant sections. Using this algorithm, individual angular curves were obtained for each cycle. Subsequently, these curves were stacked along a time axis to create a $3 \mathrm{D}$ visualization of the evolution of the data over time as well as within individual cycles. This allows the development of failures and their angular localization along the wear track to be investigated.

\section{Data evaluation}

\subsection{Outlier correction}

First, outliers were removed from the original sensor signals. Outlier removal was necessary, as regular bursts of electronic noise originating from the data acquisition system were observed in the sensor data, as shown in Fig. 2.

Outliers were detected using the inter-quartile range (IQR) method and an operating time window robust estimator of variability in the dataset. In each time window, the median and IQR were calculated, and outliers were removed from the dataset based on the following two constraints:

1) Single data points that differed by more than

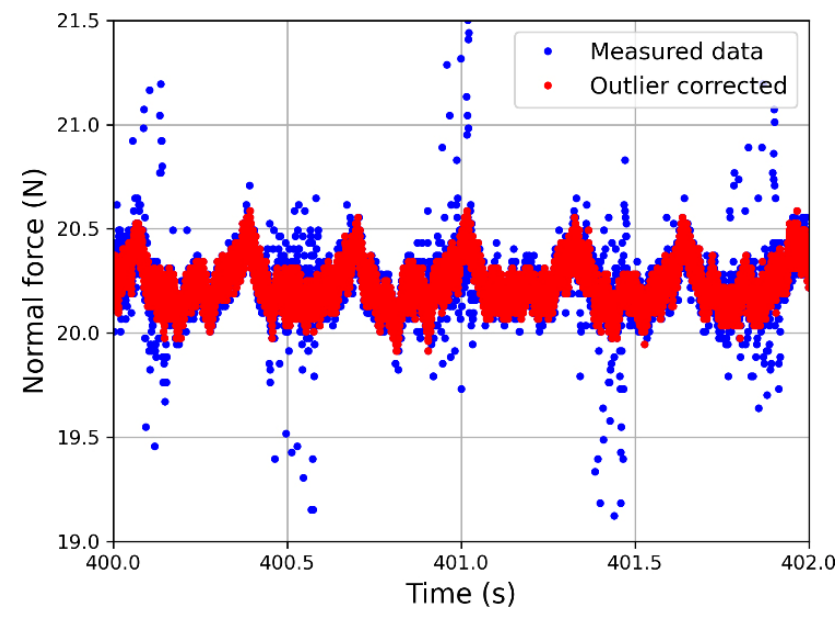

Fig. 2 Normal force data before and after outlier correction.

$n$-times the IQR from both the preceding and following points were regarded as outliers; $n$ was set to 0.5 for normal force and displacement data, and 1 for friction force data.

2) Data points that deviated by more than $3 \mathrm{IQR}$ from the median of each time window (far outliers) were removed. The factor 3 was selected to avoid clipping the top and bottom values of the signal oscillations. The first constraint was selected based on the premise that the measured physical quantities change gradually over time. Hence, a single point with a measured value clearly distinct from its vicinity can be considered as a measurement artifact rather than an actual physical effect. This criterion has been proven effective for removing random data oscillations appearing in the electronic bursts. Subsequently, the outliers were replaced by a linear interpolation between the two neighboring points. Figure 2 shows a $2 \mathrm{~s}$ segment of the normal force curves before and after outlier correction.

\subsection{TFA}

In the present study, TFA based on short-time Fourier transform (STFT) was used to reveal periodic structures in the force and displacement sensor data on short timescales, i.e., on the level of individual cycles and below, and their corresponding frequencies. Using standardized methods for pin-on-disc testing, the output may be only a single value for the investigated parameter, e.g., the average $\mathrm{CoF}$ or the total wear height. In this case, data acquisition is often performed at sampling rates below the cycle frequency of the 
tribometer, or the averages of one or several cycles are stored. However, the periodic oscillations mentioned above remained in the form of undesired noise and might significantly affect the experimental statistics.

TFA allows the localization of a signal in both the frequency and time space with reasonable accuracy by applying a sliding time window to the signal and computing the STFT in each window. It is noteworthy that STFT-based TFA is based on the uncertainty principle of signal processing, i.e., a narrow time window results in a favorable time resolution but a poor frequency resolution, and vice versa. The STFT of a function $x(t)$ at a given frequency $f$ and a given point in time $\tau$ can be written as $[23,40]$

$$
X(f, \tau)=\int_{-\infty}^{\infty} x(t) w(t-\tau) \mathrm{e}^{-j 2 \pi f t} \mathrm{~d} t
$$

where $w(t-\tau)$ is the windowing function, $x(t) w(t-\tau)$ is the time-windowed function, and $j$ is the imaginary unit with $j^{2}=-1$.

The windowing function is typically selected such that it is 1 near the origin and decays to 0 outside the window area. In the present study, a Tukey window [41] was selected as the windowing function. This window was 1 around the origin and decayed toward the window border via a cosine function.

The spectral energy density of a signal can be written as the squared amplitude of its STFT, as follows:

$$
S_{x x}(f, \tau)=|X(f, \tau)|^{2}
$$

The TFA results are shown as spectrograms. In a spectrogram, the STFT of individual time segments $\tau$ of the signal are stacked along the $x$-axis, with the frequencies shown on the $y$-axis. The values in color indicate the spectral energy density $S_{x x}(f, \tau)$ of the individual frequency components.

To obtain reasonably good resolutions in both the time and frequency domains, a time-window length of $8 \mathrm{~s}(40,000$ channels for $5 \mathrm{kHz}$ data) was selected with an overlap of $1 / 4$ of the window size between two succeeding windows. A time-window length of $8 \mathrm{~s}$ corresponded to a frequency resolution of $0.125 \mathrm{~Hz}$.

\subsection{D data visualization}

For data visualization in the form of $3 \mathrm{D}$ surface plots, the $5 \mathrm{kHz}$ sensor signals were downsampled to achieve an angular resolution of $1^{\circ}$. To avoid aliasing effects, a low-pass filter was applied prior to data reduction. In this study, a Savitzky-Golay filter [42] was used. Only every tenth cycle was used for the 3D visualization to maintain a manageable data amount, and the file size was small. The individual cycles were stacked along the $x$-axis and can therefore be considered as the time axis of the respective experiment. The angular position within the individual cycles is shown on the $y$-axis. The sensor data are presented along the $z$-axis.

The data points were transferred to a rectangular grid, and the shape of the surface between the data points was approximated via cubic interpolation in both the $x$ - and $y$-directions.

\subsection{Cycle-based data evaluation}

Periodic structures induced by the tilt and waviness of the disc contributed significantly to the variance in the sensor datasets. Sampling rates in the kilohertz range were sufficient to resolve these periodic structures throughout the experiment for rotational frequencies achievable on the pin-on-disc setup used in this study, i.e., up to $50 \mathrm{~Hz}$. As the characteristics of these oscillations were known, the apparent measurement uncertainty can be reduced by eliminating the periodic fraction of the signal for further statistical data evaluation. In this regard, the cycle means were used to describe the recorded sensor signals. Figure 3 shows a portion of the normal force data obtained from the lubricated experiment. Statistical data evaluation was performed on the outlier-corrected original dataset and the reduced dataset that contained only the cycle means. A comparison is presented in Section 4.4.

\subsection{Friction and wear}

The friction force $F_{\mathrm{f}}$ between two sliding bodies is proportional to the normal load $F_{\mathrm{N}}$ exerted on the contact and is independent of the size of the contact area. The proportionality factor $\mu$ is the CoF.

$$
F_{\mathrm{f}}=\mu \times F_{\mathrm{N}}
$$

In the present study, the $\mathrm{CoF}$ was calculated via the 


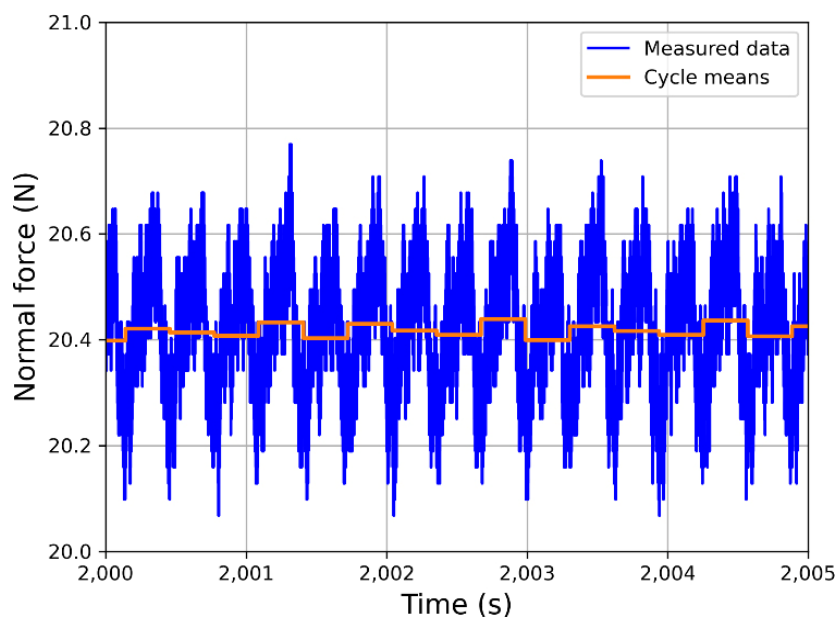

Fig. 3 Five-second time window of normal force data from lubricated experiment. Measured data (blue line) replaced by cycle means (orange line).

element-wise division of the outlier-corrected friction and normal force data. For statistical data evaluation, the first $100 \mathrm{~s}$ of each experiment were omitted to exclude instabilities in the sensor signals at the beginning of the experiment. Time-resolved histograms were constructed to visualize the temporal evolution of the CoF during the experiment. The data were partitioned into 10 equally spaced time windows. For each time window, a histogram was constructed by partitioning the data into 80 equally spaced bins. The individual histograms were stacked on top of each other, and different colors were used to indicate the corresponding time window.

Boxplots were created to compare the variability of the data within and between the individual experiments. Finally, a comparison between the $\mathrm{CoF}$ calculated from the outlier-corrected original sensor data and that based on cycle-based data evaluation was performed. The results are presented and discussed in Section 4.4.

To experimentally verify the measured wear height provided by the displacement sensor, the surface of the worn ball was investigated via light microscopy after the experiment. The diameter $d$ of the worn contact surface was measured twice, i.e., in the sliding direction and in the direction orthogonal to it. The wear area was assumed to be circular, with the mean of both measurements as the diameter.

The wear height of the ball $h_{\text {ball }}$ was approximated as follows:

$$
h_{\text {ball }}=R-\sqrt{R^{2}-r^{2}}
$$

where $R=5 \mathrm{~mm}$ is the radius of the ball and $r$ is the radius of the apparent contact surface.

The wear volume $V_{\text {ball }}$ was approximated as the segment of a sphere with a basis circle of radius $r$.

$$
V_{\text {ball }}=\frac{\pi}{6} h_{\text {ball }}\left(3 r^{2}+h_{\text {ball }}^{2}\right)
$$

The profile of the wear track on the disc was analyzed at four spots that were $90^{\circ}$ relative to each other using focus variation 3D microscopy (InfiniteFocus, Alicona Imaging $\mathrm{GmbH}$, Austria). The maximum wear depth $d_{\max }$ and cross-sectional area $A$ of the wear profile were determined. The wear volume on the disc, $V_{\text {disc }}$ was approximated by

$$
V_{\mathrm{disc}}=A \times c
$$

where $c=2 \pi r_{\text {track }}$ is the circumference of the wear track at its center with a nominal track radius $r_{\text {track }}=$ $50 \mathrm{~mm}$.

\section{Results}

\subsection{TFA}

In this subsection, spectrograms are presented and discussed based on the underlying sensor data, where frequencies below $60 \mathrm{~Hz}$ are emphasized.

A straight horizontal line in the spectrogram indicates the presence of the corresponding frequency throughout the experiment. The appearance or disappearance of frequency components throughout the experiment in the form of continuous frequency bands or discrete frequencies indicates a change in the operation mode. Short-time disturbances are depicted as continuous frequency bands with clear temporal locations.

In the dry experiments, a change in the operation mode during the experiment was observed in the spectrograms of the force sensor data. In the following, results from an exemplary experiment are discussed more comprehensively.

Figure 4(a) shows a spectrogram of the frictionforce signal. In the first 2,000 s of the experiment, a continuous frequency spectrum overlaying the discrete 
(a)

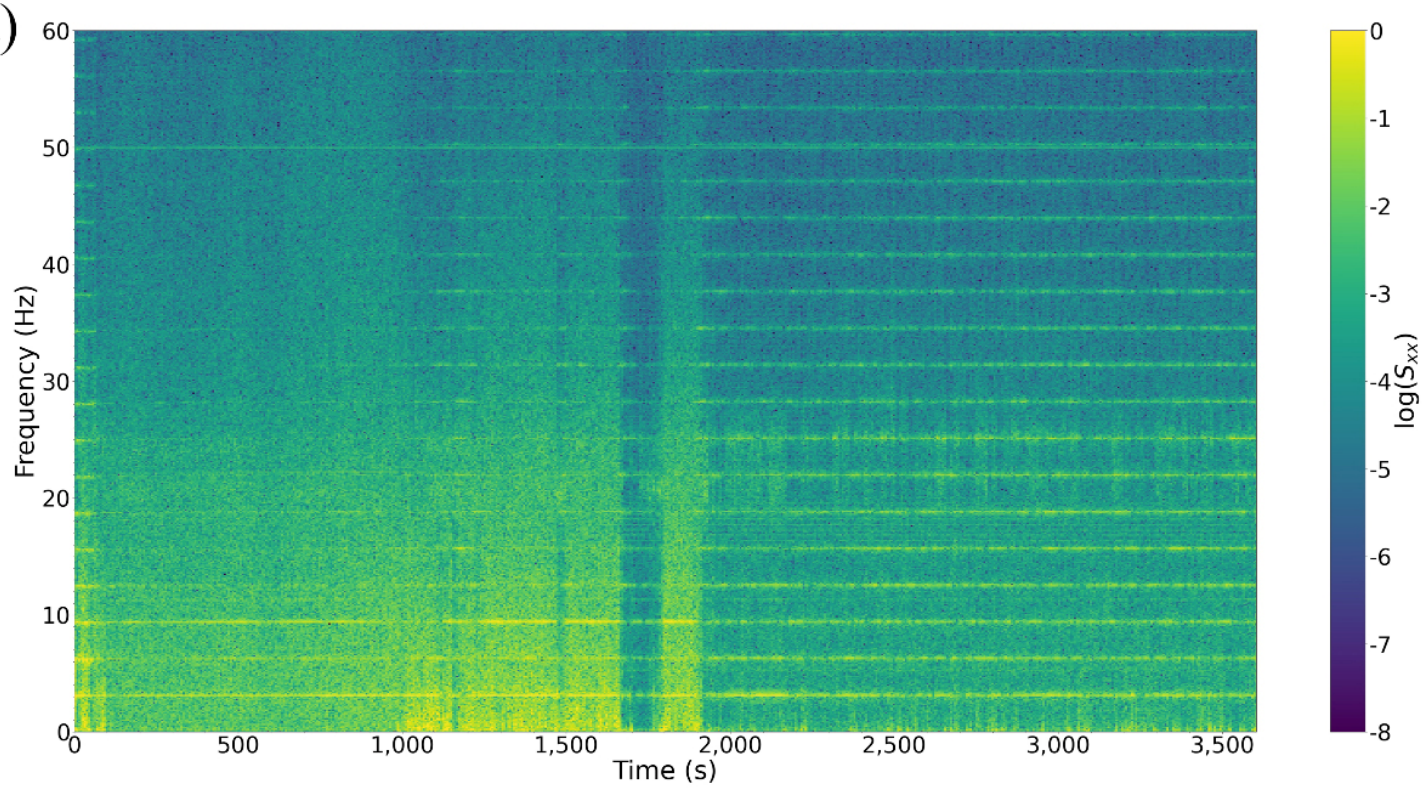

(b)

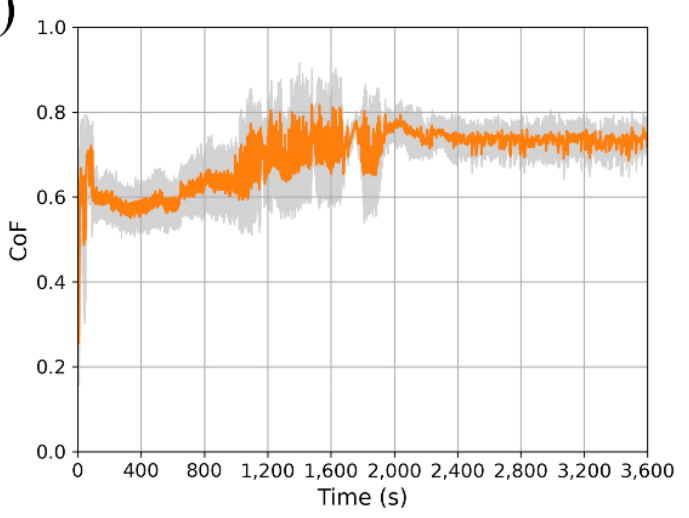

(c)

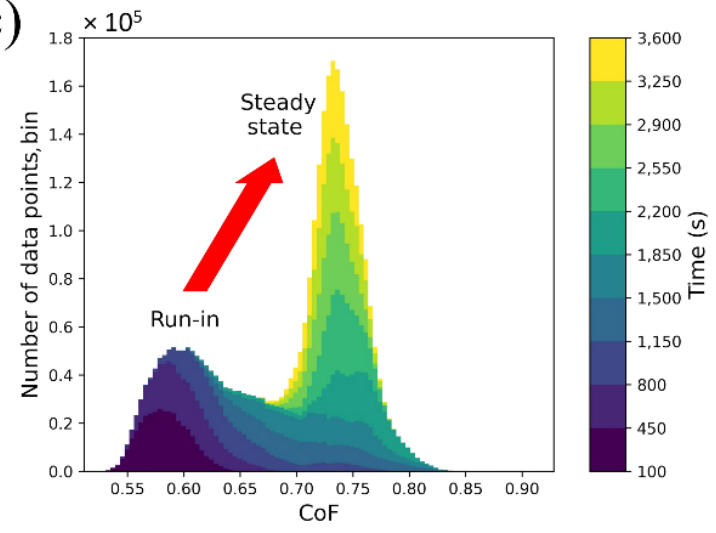

Fig. 4 (a) Spectrogram of friction force signal from dry experiment. The change in operation mode between 1,500 and 2,000 s is evident. (b) Temporal evolution of cycle means of CoF. (c) Time-resolved histogram of CoF.

line spectrum of the rotation frequency and its harmonics was visible in the spectrograms. This indicates a high level of noise in the data, which is likely due to friction-induced mechanical vibrations from the system. The variance of the measured data was higher than that at the later stage of the experiment, particularly in the time interval between 1,000 and $1,700 \mathrm{~s}$. This operation mode may be regarded as the "run-in" phase of the system. The operation mode changed after approximately 2,000 s before the system stabilized. The noise observed during the first phase was reduced significantly, and the spectrogram was dominated by the discrete frequency spectrum from the rotation frequency of the disc and its harmonics. In this mode, the operation of the tribometer stabilized, and the measured force values tended to saturate at $\sim 0.74$.

Figure 4(c) shows a time-resolved histogram of the CoF, corresponding to the curve shown in Fig. 4(b). The histogram clearly shows a bimodal distribution, where the data distributions of the individual time windows resembled Gaussian distributions, except for the transition period between 1,500 and 2,200 s. At the beginning of the experiment, the $\mathrm{CoF}$ was centered around 0.58. The data distribution shifted gradually during the change between the two operation modes. After the change, the system stabilized at a higher CoF of 0.74 .

Tribological interactions between ceramics and metal surfaces are characterized by tribochemical reactions and material transfer in the contact area. Non-oxide ceramics such as $\mathrm{Si}_{3} \mathrm{~N}_{4}$ typically form oxide layers on 
their sliding surfaces, which are derived from oxygen or water vapor in the surrounding air. On the metal surface, a tribolayer containing both $\mathrm{Fe}$ and $\mathrm{Si}$ oxides (typically $\mathrm{Fe}_{2} \mathrm{O}_{3}$ and $\mathrm{SiO}_{2}$ ) is formed [43, 44].

The scanning electron microscopy (SEM) image (acquired in backscattered electron mode - SEM-BSE to highlight material contrast, BSE means backscattered electron imaging) presented in Fig. 5 shows two distinct phases in the wear track. One phase comprised the blank disc material, whereas the other phase exhibited particulate inclusions, shown in the form of black spots (i.e., lower atomic mass). The inset in Fig. 5 shows that these inclusions appeared in clearly defined sections within the wear track. Furthermore, outside these regions, the unidirectional scratches induced by the sliding wear process were more pronounced. Energy-dispersive X-ray spectroscopy (EDX) analysis shows that the black spots were rich in oxygen and contained both Fe from the disc and $\mathrm{Si}$ from the pin (spots 1 and 2 in Fig. 5), with Fe oxides being the dominant component. Spot 3 was measured as a reference and exhibited a $\mathrm{Cr}$ content comparable to that of the bare disc material, as shown in Table 1.

These findings confirm the formation of an oxide tribolayer on the surface of the wear track owing to the tribochemical reaction and material transfer between the two bodies. Because more pronounced

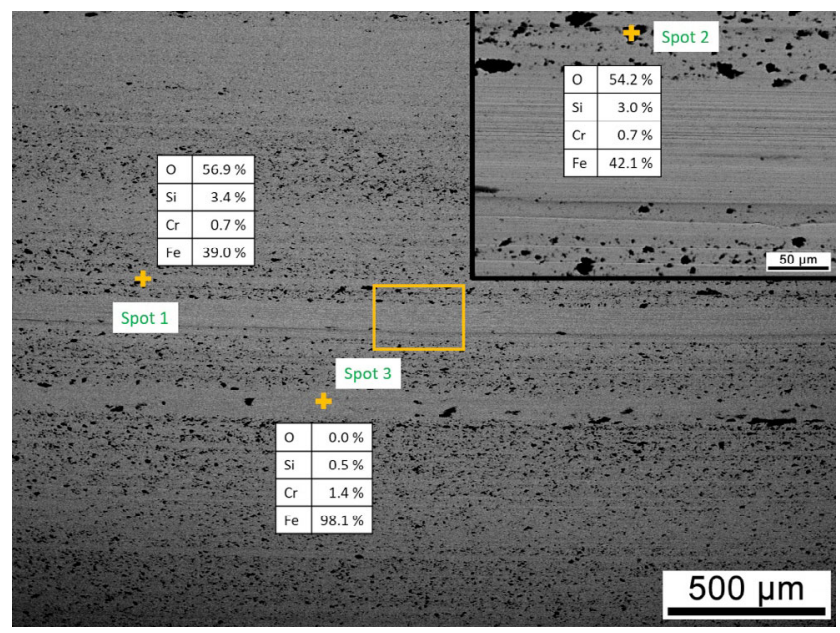

Fig. 5 SEM-BSE image with EDX results (at\%) of wear track on disc from dry experiment. High-magnification area indicated by yellow rectangle shows two clearly separated regions with and without oxide inclusions. Composition obtained from Spot 3 is comparable to that of steel substrate containing 1.35-1.60 at $\%$ Cr, according to EN ISO 683-17. wear grooves were present in regions without oxide particles, we conclude that the change in the operation state may be ascribed to the formation of an oxide layer on the surface of the wear track, thereby resulting in a stable system.

\subsection{Periodic structures in sensor data}

A 3D representation of the angle-resolved normal force signal from the lubricated experiment is shown in Fig. 6. Figure 7 shows a visualization of the displacement sensor data from the dry experiment. The colors indicate the difference between the individual data points and their corresponding cycle mean, as well as the periodic changes along the wear track.

In the dry experiments, the signal of the displacement sensor was dominated by the actual physical wear. The sensor data primarily reflected the total wear height, overlaid with the displacement along the wear track within the individual cycles. This results in gradual changes in the curve shapes, as the material was continuously removed from both the silicon nitride ball and steel disc. The total wear height was determined by calculating the mean displacement in the final $10 \mathrm{~s}$ of each experiment. An average total

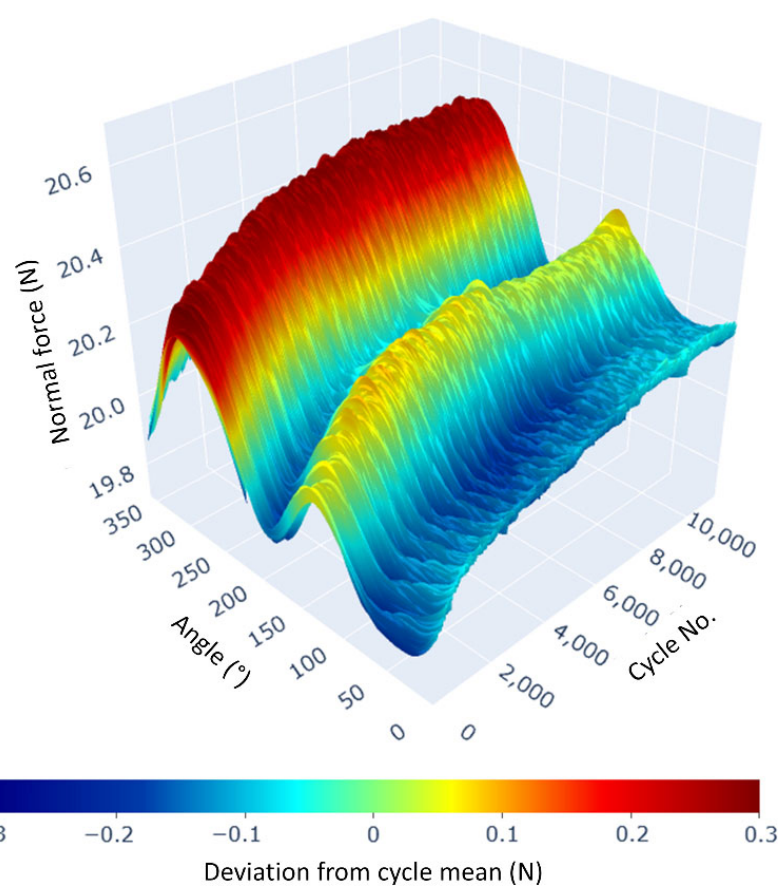

Fig. $63 \mathrm{D}$ visualization of angle-resolved temporal evolution of normal force signal from lubricated experiment. Colors indicate deviation from cycle mean. 


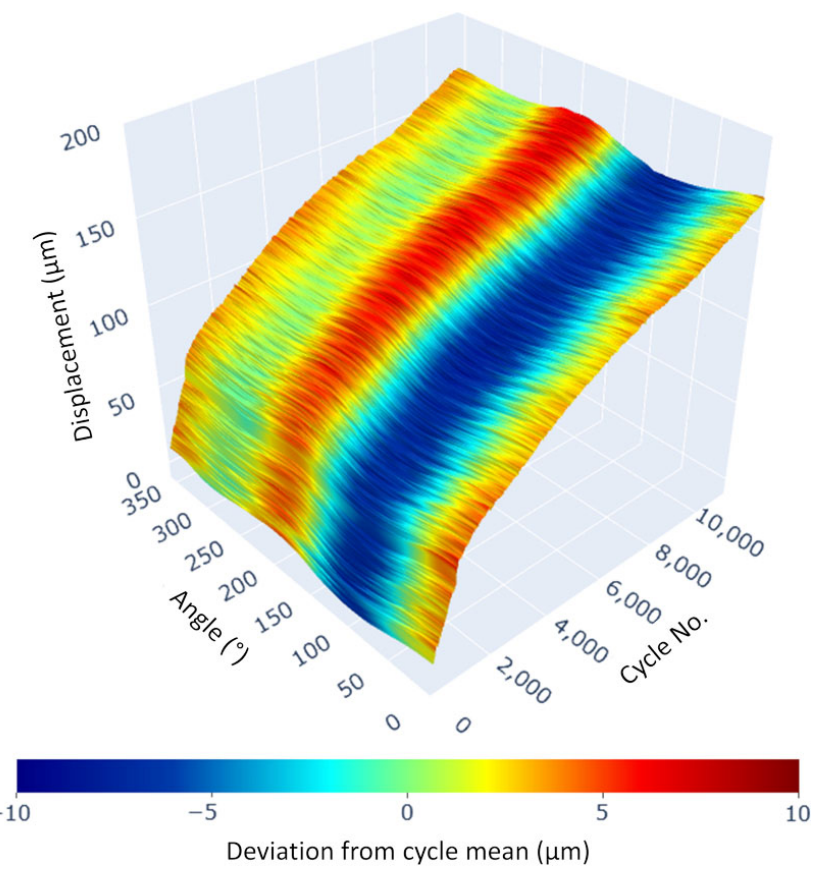

Fig. 7 3D visualization of angle-resolved temporal evolution of displacement signal from dry experiment. Colors indicate deviation from cycle mean.

wear height of $154 \pm 35 \mu \mathrm{m}$ was obtained from 10 dry experiments.

The total wear height provided by the laser displacement sensor was compared with the post-test wear measurements of the two counter bodies. The wear area of the ball was $2.275 \mathrm{~mm}$ in diameter. Hence, a wear height of $131 \mu \mathrm{m}$, corresponding to a wear volume of $0.267 \mathrm{~mm}^{3}$, was obtained.
Figure 8(a) shows a section of the wear track on the disc surface acquired using a focus variation $3 \mathrm{D}$ microscope, and the corresponding average wear profile is shown in Fig. $8(\mathrm{~b})$. The maximum depth of the wear profile $(2.46 \pm 0.18 \mu \mathrm{m})$ was obtained by averaging the four $90^{\circ}$ rotated profiles. A wear volume of $0.761 \mathrm{~mm}^{3}$ was calculated for the steel disc.

The values obtained were within the margin of deviation of the total wear height determined using the laser displacement sensor. The total wear height was dominated by wear losses on the ball. However, the wear volume of the disc was approximately three times larger than the wear volume of the ball, as the wear losses were dispersed along the circumferential wear track.

\subsection{Localized events}

The 3D visualization of the sensor data allows the detection of localized events occurring during the experiment. This includes abrupt increases in the measured data as well as individual localized events related to changes in the tribosystem, including local surface damages, break out of wear particles, or local loss of lubrication. These events can be localized in a $3 \mathrm{D}$ graph in terms of both time and angular positions.

In one of the lubricated experiments, a pronounced event that disrupted the stable operation of the system was detected. Figure 9(a) shows a 3D visualization of the $\mathrm{CoF}$ from this experiment. At three points in the
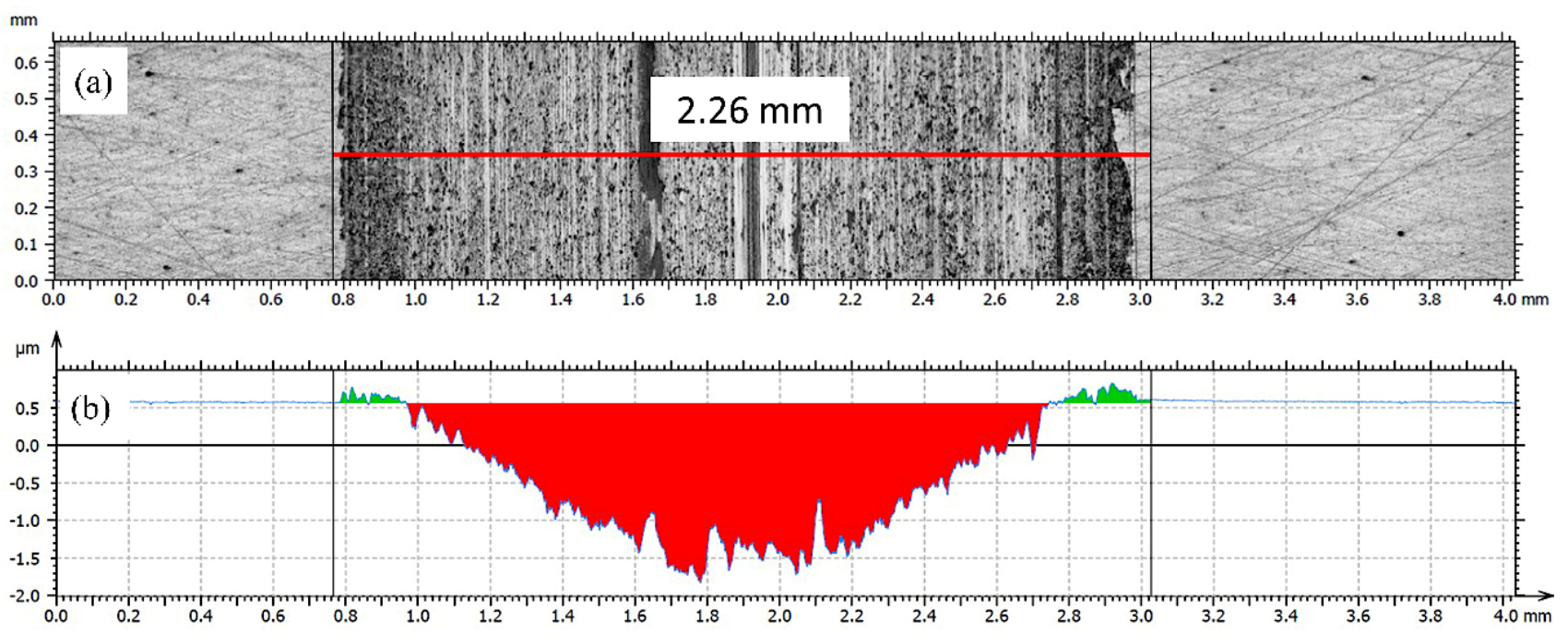

Fig. 8 (a) Microscope image of wear track section on disc surface after dry experiment. (b) Corresponding wear profile acquired along red line shown in (a). 

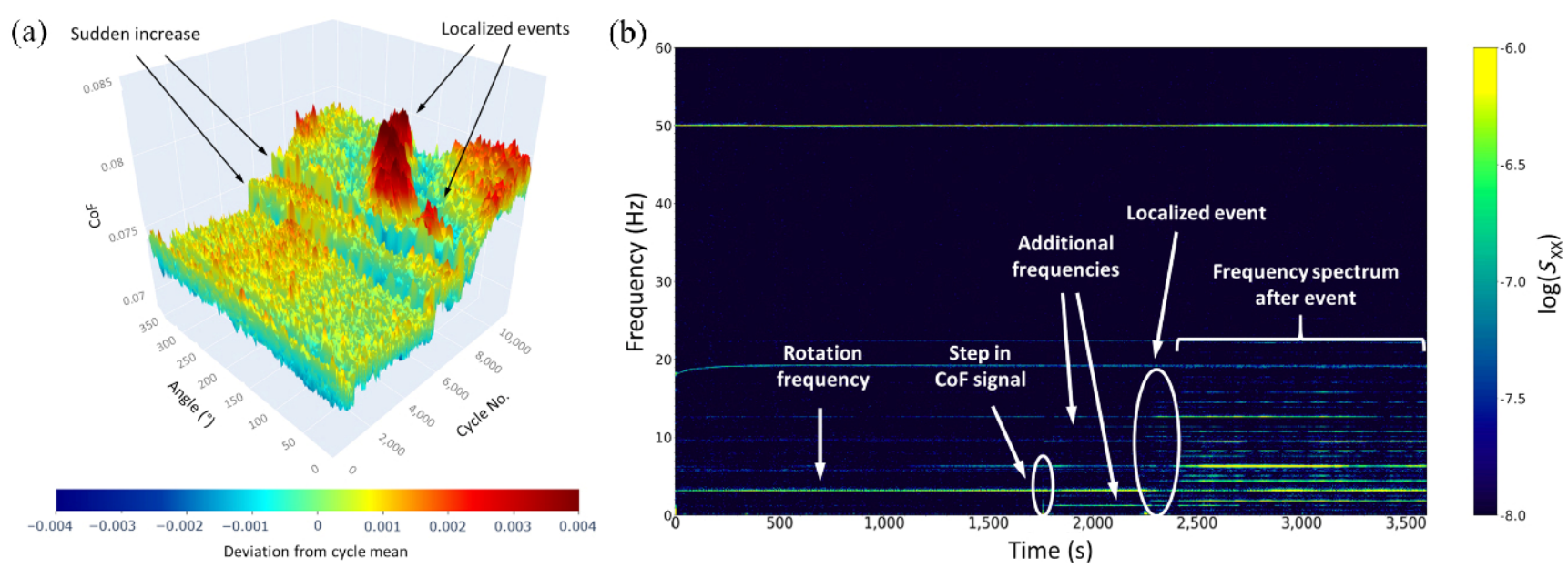

Fig. 9 (a) 3D visualization of CoF from lubricated experiment and (b) corresponding spectrogram. Short-time events are evident in 3D plot and well-resolved with respect to their temporal and angular position. Localized event after approximately $2,300 \mathrm{~s}$ results in a change in frequency spectrum.

experiment, an abrupt increase in the $\mathrm{CoF}$ due to external factors was observed. The third increase resulted in a pronounced peak that reached its maximum after approximately 8,500 cycles, with a maximum angular position at approximately $160^{\circ}$. Furthermore, this peak resulted in a change in the operating state of the system. After being nearly constant throughout the entire experiment, except for the two abrupt increases, which are visible as steps in the time-series data, the CoF began increasing slowly. The angular dependence of the $\mathrm{CoF}$ became more pronounced at values exceeding 0.08 , which were observed near the $0^{\circ}$ position on the wear track toward the end of the experiment, as shown in Fig. 9(a). A CoF of 0.081 was achieved at the peak maximum.

The state change observed in the time-series data of the CoF, as visualized in Fig. 9(a), is similarly presented in the corresponding spectrogram, as shown in Fig. 9(b). The abrupt increase in the time-series data at approximately $1,800 \mathrm{~s}$ can be identified as a faint vertical line in the low-frequency range and resulted in additional frequencies, which appeared most prominently at approximately one third of the rotation frequency (i.e., approximately $1 \mathrm{~Hz}$ ) and slightly below the third harmonic (i.e., approximately $11 \mathrm{~Hz}$ ). The localized event, which occurred at approximately 2,400 s, resulted in multiple additional discrete frequencies in the range between 0 and $20 \mathrm{~Hz}$. These frequencies were recorded in the spectrogram until the end of the experiment.

\subsection{Cycle-based data evaluation}

Figure 10 show box plots of the $\mathrm{CoF}$ from the 10 lubricated and 10 dry experiments. The range of the boxes is between the $25 \%$ and $75 \%$ quantiles, and the horizontal line indicates the median.

The whiskers extend between the minimum and maximum values of each dataset. Asymmetries in the boxplots indicate changes in the operation state during the experiment. The light boxes in Fig. 10 show
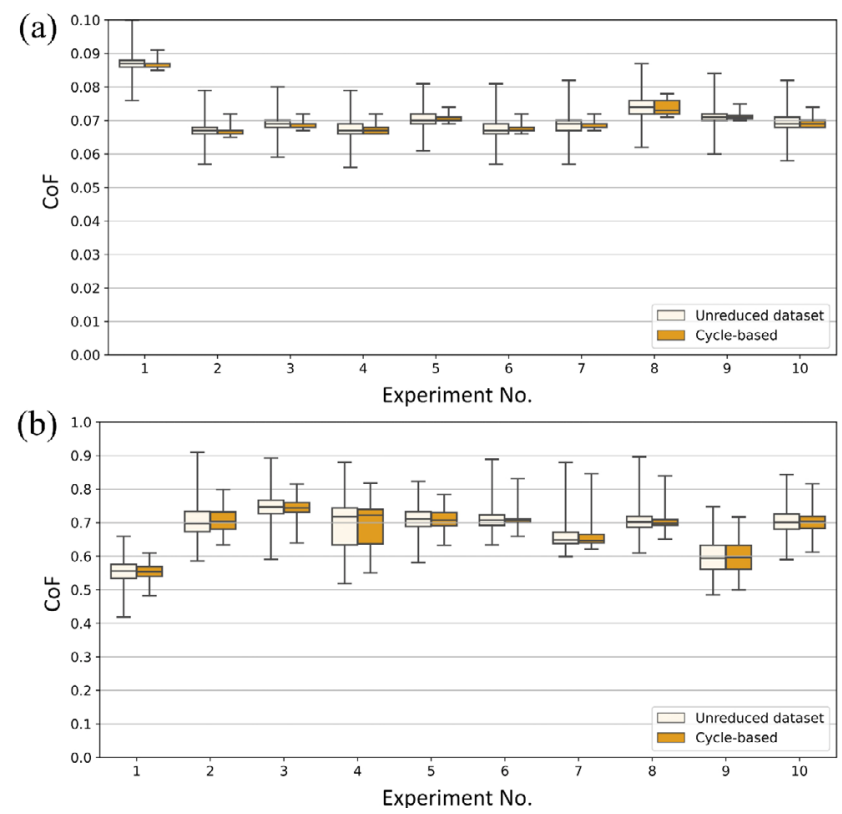

Fig. 10 Box plots of CoF from (a) 10 lubricated experiments and (b) 10 dry experiments. 
the results obtained using the unreduced outliercorrected dataset. This evaluation method is labeled "conventional" in the graphs. The dark boxes, labeled "cycle-based", show the results obtained using the reduced dataset that contained only the cycle means.

The results of the lubricated experiments indicated low variability. The median $\mathrm{CoF}$ was approximately 0.07, except for Experiment No.1, whose CoF was slightly higher. A comprehensive discussion of Experiment No. 8 is provided in the previous section. As the lubricated experiments showed a pronounced cyclic behavior, i.e., the difference between the minima and maxima, the apparent measurement uncertainty in the reduced dataset was considerably smaller than that in the unreduced data. The medians and box positions were only slightly affected by data reduction, with the box heights being generally smaller for the reduced data.

The effect of cycle-based data evaluation is generally less pronounced in dry experiments, as considerable sections of each experiment are dominated by frictioninduced vibrations that dominate the force sensor data. However, the medians and box positions were comparable for both evaluation methods. The $\mathrm{CoF}$ indicated a higher variability, i.e., approximately 0.7 , in most of the experiments. A comprehensive discussion of Experiment 4 is provided in Section 4.1. The CoF values obtained were comparable to values pertaining to experiments using a pin/ball-on-disc setup with a silicon nitride on metal contact $[45,46]$.

\section{Discussion}

Comprehensive analyses of high-speed sensor data from tribological experiments performed in this study revealed periodic structures, which remain hidden in standard acquisitions of sensor data at certain time intervals or in averaged data [17]. This will consequently impede the measurement accuracy, and localized events may be completely hidden.

These periodic structures have been observed on pin-on-disc setups $[15,16]$ but have been disregarded in most tribology studies. Additionally, some newer commercial oscillating tribometers allow the recording of sensor data with a high sampling rate over a limited duration to investigate the structure of individual strokes [22]. Nevertheless, standard tribometers output cycle averages for the $\mathrm{CoF}$ in normal operations, whereas raw data are typically not stored and hence not available for post-processing [17]; consequently, useful additional information is discarded.

However, current developments pertaining to the storage and handling of large datasets permit the acquisition and efficient storage of data at high sampling rates during a complete (long-term) experiment, which may require several hours or even days. Online or post-test treatments of these large datasets can reveal hidden information regarding tribological interactions.

In the present study, it was demonstrated that STFT-based TFA is effective for detecting periodic structures and their underlying frequencies, as well as changes in the operation mode of the tribological system based on the recorded force and displacement sensor data. This represents a significant improvement compared with the typical interpretation of the (averaged) $\mathrm{CoF}$ evolution over time.

Segmenting the measured data into single cycles and stacking them along a time axis allows one to investigate changes during the experiment. This promotes the detection of short-time events during an experiment, whose source can be localized on the disc surface via its angular position. This procedure is extremely effective compared with using standard CoF curves (or other tribological time-evolution data). Standard CoF curves often result in difficult-tointerpret abrupt "random" peaks or valleys when local instabilities (e.g., wear particles or oxides) cause changes in the CoF. In particular, when data recording is performed at time intervals that are not synchronous with the cycle period, a friction value that is extremely high or low may be obtained, or aliasing effects or oscillations may be yielded, thereby resulting in an artifact in data acquisition or averaging.

Cycle-based statistical data evaluation using cycle means results in a significant reduction in the apparent measurement uncertainty. Therefore, the evaluation of data from pin-on-disc setups becomes more reliable and precise, as the periodic patterns caused by disc tilt and waviness are eliminated and any noise present in the data is reduced significantly. In addition, the original dataset remains available for a detailed investigation of events with subcycle resolution, which may subsequently be used to localize the position failures precisely during post-test evaluation, e.g., 
via SEM or other methods of surface analytics.

A set of data analysis and visualization methods enabled by high-frequency data acquisition was presented herein. This workflow can be utilized to observe other tribological systems comprehensively. We performed a triboexperiment with a static load and velocity. We envision that experiments with dynamic changes, cycle-based, or localized measurements, and depiction of tribological data will become more important.

\section{Conclusions}

High-speed data acquisition was performed to investigate the dynamic behavior of force and displacement sensor data obtained from on a pin-on-disc setup. In summary, the main conclusions of this study are as follows:

1) The sensor data obtained from pin-on-disc setups exhibited periodic structures, which can be revealed by the presented workflow.

2) STFT-based TFA showed that the rotation frequency of the disc was $3.18 \mathrm{~Hz}$, and its harmonics were the dominant frequencies in the sensor signals. Changes in the friction regime can be localized precisely in the frequency data.

3) Acquisition frequencies that were sufficiently high to resolve periodicities enabled cycle-based data evaluation, revealing localized changes in CoF.

4) Cycle-based data evaluation together with STFTbased TFA and 3D visualization techniques enabled the detection of localized events as well as changes in the operation state.

5) Data reduction based on cycle means significantly reduced the apparent measurement uncertainty for datasets containing periodic structures.

The presented data evaluation workflow of highspeed tribological data can be used for other tribological test setups and may reveal tribological phenomena that are concealed by the measurement noise. The methods are highly promising, particularly when used in dynamic setups.

\section{Acknowledgements}

This work was funded by the Austrian COMET
Program (Project InTribology, No. 872176) via the Austrian Research Promotion Agency (FFG) and the Provinces of Niederösterreich and Vorarlberg, and has been carried out within the Austrian Excellence Centre of Tribology (AC2T research $\mathrm{GmbH}$ ).

Open Access This article is licensed under a Creative Commons Attribution 4.0 International License, which permits use, sharing, adaptation, distribution and reproduction in any medium or format, as long as you give appropriate credit to the original author(s) and the source, provide a link to the Creative Commons licence, and indicate if changes were made.

The images or other third party material in this article are included in the article's Creative Commons licence, unless indicated otherwise in a credit line to the material. If material is not included in the article's Creative Commons licence and your intended use is not permitted by statutory regulation or exceeds the permitted use, you will need to obtain permission directly from the copyright holder.

To view a copy of this licence, visit http://creativecommons.org/licenses/by/4.0/.

\section{References}

[1] Cabboi A, Woodhouse J. Validation of a constitutive law for friction-induced vibration under different wear conditions. Wear 396-397: 107-125 (2018)

[2] Cabboi A, Woodhouse J. Identifying short-term variation of dynamic friction by means of its frequency response function. J Sound Vib 472: 115212 (2020)

[3] Becker S, Popp U, Greiner C. A reciprocating optical in situ tribometer with high-speed data acquisition. Rev Sci Instrum 87(8): 085101 (2016)

[4] US-ASTM. ASTM G99 Standard test method for wear testing with a pin-on-disk apparatus. ASTM, 2000.

[5] ISO. ISO 7148-1:1999 Plain bearings-Testing of the tribological behaviour of bearing materials-Part 1: Testing of bearing metals. ISO, 2001.

[6] ISO. ISO 7148-2:1999 Plain bearings - Testing of the tribological behaviour of bearing materials-Part 2: Testing of polymer-based bearing materials. ISO, 2001.

[7] Ahmer S M H, Jan L S, Siddig M A, Abdullah S F. Experimental results of the tribology of aluminum measured with a pin-on-disk tribometer: Testing configuration and additive effects. Friction 4(2): 124-134 (2016) 
[8] Uflyand I E, Zhinzhilo V A, Burlakova V E. Metalcontaining nanomaterials as lubricant additives: State-of-the-art and future development. Friction 7(2): 93-116 (2019)

[9] Zhang X A, Zhao Y Z, Ma K, Wang Q. Friction behavior and wear protection ability of selected base lubricants. Friction 4(1): 72-83 (2016)

[10] Riva G, Varriale F, Wahlström J. A finite element analysis (FEA) approach to simulate the coefficient of friction of a brake system starting from material friction characterization. Friction 9(1): 191-200 (2021)

[11] Prajapati D K, Tiwari M. The correlation between friction coefficient and areal topography parameters for AISI 304 steel sliding against AISI 52100 steel. Friction 9(1): 41-60 (2021)

[12] Blutmager A, Spahn T, Varga M, Friesenbichler W, Riedl $\mathrm{H}$, Mayrhofer P H. Processing fiber-reinforced polymers: Specific wear phenomena caused by filler materials. Polym Eng Sci 60(1): 78-85 (2020)

[13] Rodríguez Ripoll M, Scheichl B, Bianchi D, Jakab B, Franek F. Development of a mechanical model of doctor blade-press roll tribosystem with aim to optimise cleaning performance: Numerical predictions and first experimental verification. Tribol Mater Surf Interfaces 8(1): 41-47 (2014)

[14] Aronov V, D'Souza A F, Kalpakjian S, Shareef I. Experimental investigation of the effect of system rigidity on wear and friction-induced vibrations. J Lubric Technol 105(2): 206211 (1983).

[15] Godfrey D. Friction oscillations with a pin-on-disc tribometer. Tribol Int 28(2): 119-126 (1995)

[16] Hsiao E, Kim S H. Analyzing periodic signals in rotating pin-on-disc tribotest measurements using discrete Fourier transform algorithm. Tribol Lett 35(2): 141-147 (2009)

[17] Balarini R, Diniz G A S, Profito F J, Souza R M. Comparison of unidirectional and reciprocating tribometers in tests with MoDTC-containing oils under boundary lubrication. Tribol Int 149: 105686 (2020)

[18] Larsson R. Modelling the effect of surface roughness on lubrication in all regimes. Tribol Int 42(4): 512-516 (2009)

[19] Jin L, Scheerer H, Andersohn G, Oechsner M, Hellmann D. Experimental study on the tribo-chemical smoothening process between self-mated silicon carbide in a water-lubricated surface-contact reciprocating test. Friction 7(2): 181-191 (2019)

[20] Vengudusamy B, Grafl A, Novotny-Farkas F, Schöfmann W. Comparison of frictional properties of gear oils in boundary and mixed lubricated rolling-sliding and pure sliding contacts. Tribol Int 62: 100-109 (2013)
[21] Blau P J. The significance and use of the friction coefficient. Tribol Int 34(9): 585-591 (2001)

[22] Patzer G, Shah R, Schneider A, Iaccarino P. New approach to interpreting seizure tests on the translatory oscillation tribometer (SRV). Lubricants 7(11): 93 (2019)

[23] Cohen L. Time-frequency distributions-A review. Proc IEEE 77(7): 941-981 (1989)

[24] Sejdić E, Djurović I, Jiang J. Time-frequency feature representation using energy concentration: An overview of recent advances. Digit Signal Process 19(1): 153-183 (2009)

[25] Yang Y, Peng Z K, Zhang W M, Meng G. Parameterised time-frequency analysis methods and their engineering applications: A review of recent advances. Mech Syst Signal Process 119: 182-221 (2019)

[26] Jáuregui J C, Reséndiz J R, Thenozhi S, Szalay T, Jacsó Á, Takács M. Frequency and time-frequency analysis of cutting force and vibration signals for tool condition monitoring. IEEE Access 6: 6400-6410 (2018)

[27] Sun J L, Wang R H, Duan H F. Multiple-fault detection in water pipelines using transient-based time-frequency analysis. J Hydroinform 18(6): 975-989 (2016)

[28] Feng Z P, Liang M, Chu F L. Recent advances in timefrequency analysis methods for machinery fault diagnosis: A review with application examples. Mech Syst Signal Process 38(1): 165-205 (2013)

[29] Eder S J, Bianchi D, Neacşu I A, Vorlaufer G. An experimental and signal analysis workflow for detecting cold-induced noise emissions (cold squealing) from porous journal bearings. Mech Syst Signal Process 115: 60-69 (2019)

[30] Menon A K, Boutaghou Z E. Time-frequency analysis of tribological systems-Part I: Implementation and interpretation. Tribol Int 31(9): 501-510 (1998)

[31] Menon A K, Boutaghou Z E. Time-frequency analysis of tribological systems-Part II: Tribology of head-disk interactions. Tribol Int 31(9): 511-518 (1998)

[32] Song J, Liu T, Shi H Y, Yan S Z, Liao Z H, Liu Y H, Liu W Q, Peng Z X. Time-frequency analysis of the tribological behaviors of $\mathrm{Ti}_{6} \mathrm{Al}_{4} \mathrm{~V}$ alloy under a dry sliding condition. J Alloys Compd 724: 752-762 (2017)

[33] ISO. ISO 683-17:2014 Heat-treated steels, alloy steels and free-cutting steels-Part 17: Ball and roller bearing steels. ISO, 2014.

[34] Information. https://www.hbm.com/en/4561/ly-linear-straingauges-with-1-measurement-grid/, 2020.

[35] Information. https://www.keyence.co.uk/products/sensor/ laser/il/models/il-030/, 2020.

[36] Information. https://www.hbm.com/en/2644/clip-analogelectronics-for-industrial-measurement-tasks/, 2020. 
[37] Information. https://www.ni.com/en-us/support/model.pci6221.html, 2020.

[38] Shannon C E. Communication in the presence of noise. Proc IRE 37(1): 10-21 (1949)

[39] Information. https://www.hdfgroup.org/solutions/hdf5/, 2020.

[40] Vio R, Wamsteker W. Joint time-frequency analysis: A tool for exploratory analysis and filtering of non-stationary time series. Astron Astrophys 388(3): 1124-1138 (2002)

[41] Harris F J. On the use of windows for harmonic analysis with the discrete Fourier transform. Proc IEEE 66(1): 51-83 (1978)

[42] Savitzky A, Golay M J E. Smoothing and differentiation of data by simplified least squares procedures. Anal Chem 36(8): 1627-1639 (1964)

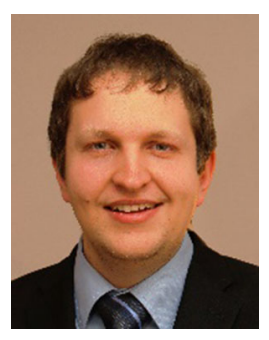

Josef PROST. He received his Ph.D. degree in physics from Vienna University of Technology, Austria, in 2018. He has been working as a scientist at the Austrian Excellence Centre for Tribology (AC2T research $\mathrm{GmbH}$ ) in Wiener Neustadt, Austria,

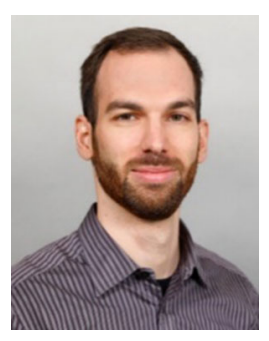

Guido BOIDI. He is a scientist at $\mathrm{AC} 2 \mathrm{~T}$ research $\mathrm{GmbH}$ (Austria) since 2019. He received his Ph.D. degree from the University of São Paulo (Brazil) in 2019, where he studied the tribological effect of surface irregularities (laser texturing

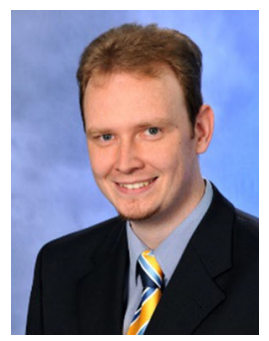

Thomas LEBERSORGER. He is a scientist at AC2T research $\mathrm{GmbH}$ (Austria) since 2004, where he is head of the department "Tribosystem Characterisation". He received his Dipl.-Ing. (FH) degree in mechatronics and microsystems
[43] Kalin M, Vižintin J, Novak S, Dražič G. Wear mechanisms in oil-lubricated and dry fretting of silicon nitride against bearing steel contacts. Wear 210(1-2): 27-38 (1997)

[44] Hutchings I, Shipway P. Tribology: Friction and Wear of Engineering Materials. 2nd edn. Oxford (UK): John Wiley \& Sons, 2017.

[45] Wang L, Wood R J K, Harvey T J, Morris S, Powrie H E G, Care I. Wear performance of oil lubricated silicon nitride sliding against various bearing steels. Wear 255(1-6): 657-668 (2003)

[46] Zhao B, Khader I, Raga R, Degenhardt U, Kailer A. Tribological behavior of three silicon nitride ceramics in dry sliding contact against Inconel 718 over a wide range of velocities. Wear 448-449: 203206 (2020)

since 2019. His main research interest is the application of advanced data analysis and visualization methods to tribological research questions, including multivariate statistical analysis as well as classification of the operation state and anomaly detection using machine learning models.

and porosity in sintered materials) under the guidance of Prof. Izabel Fernanda MACHADO. He was a visitor Ph.D. student for one year at Imperial College London (2018-2019) under the guidance of Prof. Daniele DINI. His research interests involve surface texturing, tribology of powder metallurgy, spark plasma sintering, and sintered bearings and gears.

in 2005 and his M.Sc. degree in tribology and surface engineering in 2013 from the University of Applied Sciences Wiener Neustadt. He has a broad experience in designing customer-orientated procedures for tribological tests. His research interests include rolling contact fatigue in rail/wheel contacts and data analysis. 


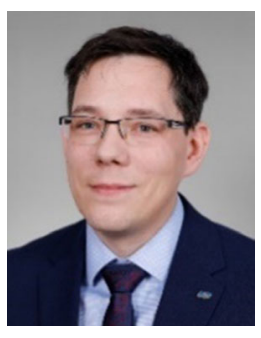

Markus VARGA. He is currently leading the strategic research area "Synaptic Tribology" at the Austrian Excellence Centre for Tribology (AC2T research $\mathrm{GmbH}$ ). He received his M.Sc. degree at the University of Applied Science Wiener Neustadt,

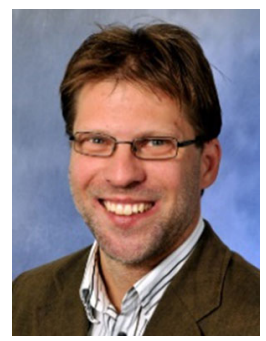

Georg VORLAUFER. He is currently a principal scientist at AC2T research $\mathrm{GmbH}$, the Austrian Excellence Centre for Tribology. He completed his M.S. degree in physics at Vienna University of Technology, Austria, in 1998 and received his Ph.D. degree in physics in 2002 from the same institution. Between 1998 and 2001, he carried
Austria, in mechatronics and completed his Ph.D. degree in tribology at the Montanuniversität Leoben, Austria. His main research field is the optimization of industrial maintenance by tribological measures for more than 10 years, i.e., wear protection, sensors for early detection of failures.

out his Ph.D. studies in the field of vacuum and surface science at CERN (Geneva, Switzerland). He has more than 18 years of experience in the field of tribology. Although since many years his research interests have been mainly in the field of physicsbased modelling and simulation of tribological systems, he is currently concentrating on tribology-related aspects of data science, machine learning, and artificial intelligence. 